http://jmscr.igmpublication.org/home/ ISSN (e)-2347-176x ISSN (p) 2455-0450

crossref DOI: https://dx.doi.org/10.18535/jmscr/v7i10.61

\author{
Dournal Of Medical Science And Clinical Research \\ IGM Publication \\ An official Publication of IGM Publication
}

\title{
An Interesting Case of Secondary Post Partum Haemorrhage
}

\author{
Authors \\ Dr Sowmya Varudu ${ }^{1}$, Dr Renuka Devi ${ }^{2}$, Dr R. Jayanthi ${ }^{3}$ \\ ${ }^{1}$ Junior Resident, ${ }^{2}$ Professor \\ MMCHRI
}

\section{Introduction}

Secondary postpartum haemorrhage $(\mathrm{PPH})$ is defined as bleeding from or into the genital tract 24 hours after the birth of the baby within 6 weeks postpartum. It affects only 1-2\% of postnatal women. This low incidence of secondary PPH and its linkage to maternal morbidity rather than mortality was the reason for the little attention among obstetricians, but it is recently gaining importance and interest with the increasing morbidity and mortality. ${ }^{(1)}$ Globally PPH remains the leading cause of maternal deaths. However in many low income countries (which accounts for highest burden) like India, there is still scarcity of information on magnitude of and risk factors for $\mathrm{PPH}$. The jeopardy of PPH is rising with the secondary form of $\mathrm{PPH}$, when women are already discharged home.PPH is widely underestimated obstetric problem with variable occurrence and severity when diagnosed. The most common aetiological factors are retained products of conception and sub involution of placental site/ endometritis $^{(1)(6)}$. A rare cause is partial or complete dehiscence of lower uterine segment incision $^{(8)}$, placental site trophoblastic tumour or choriocarcinoma.

\section{Case Report}

Mrs. X. 26 years old, P2L2 was admitted in our hospital on $09 / 11 / 2018$ ( $15^{\text {th }}$ post-op day) with the history of repeat c-section done at private hospital with an indication being PPROM (membrane ruptured outside) was discharged on third postoperative day with the instruction to come for suture removal on eight post op day, she went for suture removal and was found to have wound infection, hence she was referred to our hospital, no other specific complaints or comorbidities . On Examination patient was afebrile and mild pallor present. Systemic Examination: CVS and RSfound to be normal.

Abdominal examination: Pfannenstiel scar with intact sutures, with foul smelling purulent discharge with in duration.

Per-vaginal examination: uterus found to be 8 to 10 weeks, firm, os patulous, minimal bleeding per vaginum present, for nices free. Patient was admitted for the treatment of c-section wound infection with anaemia. Vitals were checked and found to be normal, basic blood investigations, pus for culture and sensitivity were sent. Sutures were removed and sterile Betadine dressing was done. Oral antibiotics T. Taxim O200mg BD and T. Metronidazole 400mg BD was started. 
On $4^{\text {th }}$ day of admission, suddenly at mid-night patient had a bout of vaginal bleeding. On Examination patient was conscious, responding to verbal comments, looking pale. Pulse was 100 bpm, BP was 70/50mmhg, LSCS wound in duration present, mild abdominal distention and diffuse tenderness over the suprapubic region, uterus found to be 16 to 18 weeks size, blood clots about 700 grams with copper - $\mathrm{T}$ was expelled, there was soaking of the bed sheet, bleeding $\mathrm{P} / \mathrm{V}+$, vagina was packed with 2 mop-pads, haemaccel was started followed by dopamine drip, Blood bank was alerted to issue 4 units of whole blood. Bedside ultrasound shows there is no hemoperitoneum but collection of blood in the uterus present. After fluid replacement and dopamine, BP was 120/80mmHg, PR 120 bpm, SPO2 was $99 \%$, RR was 24/min CVS SIS2normal, RS clear. Consent taken for laparotomy and proceed with an indication being secondary PPH due to? sepsis, and patient was shifted to the theatre with lower limbs in flexed position to improve the venous return. In theatre the vaginal pad along with 250 grams of clots were removed, bleeding from the cervical os present.

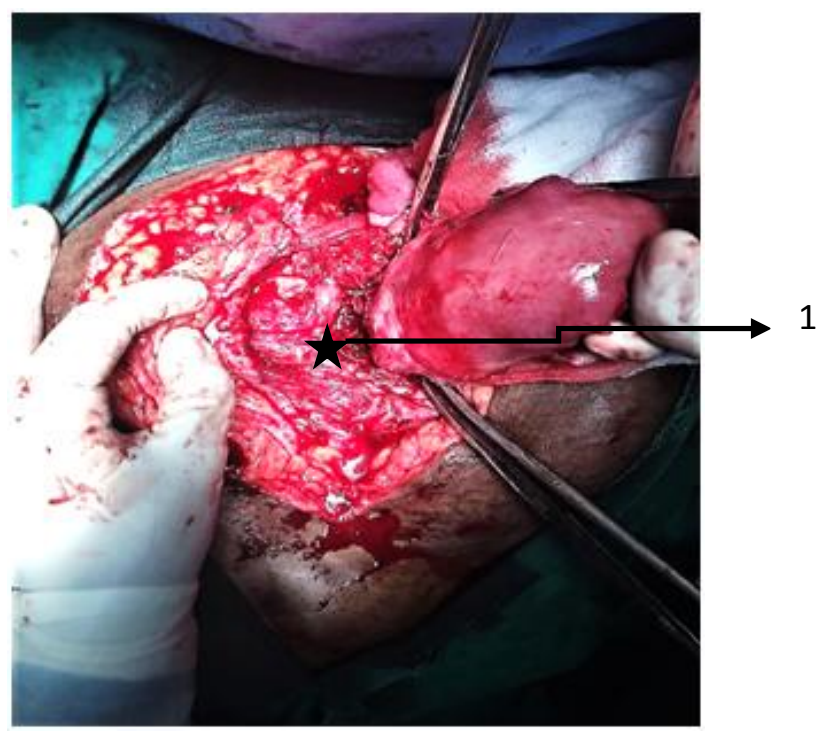

Figure 1: bladder along with peritoneum separated from upper part of uterine incision

On Laparotomy Uterus 16 to 18 weeks, flabby, bladder was densely adherent to the uterus. As there was difficulty in separating the bladder, Urologist was called in. It was found that Bladder was sutured to the anterior wall of the upper uterine segment, same released. Lower segment of uterus identified below the bladder which was caught with an allice forceps. As the placental bed was unhealthy, proceeded with subtotal hysterectomy and the specimensent for HPE.3 units of blood and 3 FFP's were given intraoperatively. Patient was given 4 units packed cells post operatively, she recovered well and repeat haemoglobin was found to be $10.2 \mathrm{~g} \%$ (after 7 units of blood and 3 FFP's). Post-operative period uneventful, sutures were removed on 8 post op day, patient was on continuous bladder drainage for 21days and discharged on 24th postop day.

Histopathology report: large dilated and thrombosed blood vessels were seen, indicating PPH due to sub involution of uterus. She was reviewed 2 weeks after surgery, general condition was good and wound healed well. This case is an example of wrong technique along with sepsis, is the cause for secondary PPH.

\section{Discussion}

PPH is the leading cause of morbidity and mortality among pregnant ladies throughout the world causing 140,000 deaths each year globally, this corresponds to the one woman dying in every 4 minutes and it is the $5^{\text {th }}$ most common cause of maternal mortality throughout the world.

\section{Conclusion}

This is a case of improper technique with endometritis.

If a stay suture is done in the lower segment, during the caesarean section along with proper antibiotics this complication could have been prevented.

Prevention of secondary PPH: Prophylactic antibiotic within 1 hour of surgery and adequate post -operative antibiotics coverage. Check for the placental cotyledons and placental membranes were removed in toto. Adequate uterotonics 
should be administered to ensure good uterine tone before closure of abdomen. Vigilant monitoring of vitals and bleeding P/V for 24 to 48 hrs post-delivery, watch for sepsis- persistent fever of $38 \mathrm{c}$ taken 4 hours apart after 24 hours of delivery, tachycardia, purulent or offensive vaginal discharge. If abnormal discharge $\mathrm{P} / \mathrm{V}$ or from the wound site send for $\mathrm{C} / \mathrm{S}$ and treat appropriately. Involution of the uterus to be checked.

\section{Management}

Approximately $10 \%$ of cases present with massive haemorrhage, require immediate resuscitation and establish the cause by speculum examination and exploration of the uterus. (Intravenous fluids, plasma expanders, blood and blood products, 10 to $15 \mathrm{~L} / \mathrm{min}$ oxygen should be given and close monitoring on vitals). Investigations: Baseline blood tests should include full blood count, coagulation profile, C-reactive protein and serum beta HCG. vaginal swab and swab from wound and mid-stream urine for culture if signs of infection suspected. Ultrasound imaging, duplex colour Doppler of pelvis if retained products suspected. Additional Imaging for specific causes (chest film, CT scanning for metastasis in choriocarcinoma, MRI for placenta accrete and angiography for interactable bleeding of unknown origin). Treatment: Antibiotics; uterine evacuation, if not responding hysterectomy is the treatment of choice for secondary PPH for sepsis (Hysterectomy carries significant risk but it is life saving and should be considered early rather than late in cases of massive haemorrhage. If delayed, patient in shock will go for DIC/acidosis, all add, operative risk and may contribute to its failure. Uterotonics, bilateral uterine artery embolization and selective arterial embolization, arterial ligation for sub involution in young women contemplating pregnancy.

\section{References}

1. Hoveyda F, Mac Kenzie IZ. Secondary $\mathrm{PPH}$ incidence, morbidity and current management. BJOG 2001:108:927-30

2. Alexander J, Thomas PW, Sanghera J. Treatments for secondary PPH (Review). Conchrane Database Syst Rev 2008;

3. Sheldon WR, Blum J, Vogel JP, Souza JP, Gulmezoglu AM, Winikoff B. Postpartum haemorrhage management, risks and maternal outcomes: findings from $\mathrm{WHO}$ multicounty survey on maternal and new born health.BJOG.2014;121Suppl 1:5-13.

4. Kittur S, Swetha D. Emergency peripartum hysterectomy-a study in tertiary care centreand medical college in Hubli, North Karnataka,India.Int J Reprod Contracep ObstetGynaecol.2016:5:1097-11013

5. Shirodker SD, Pandey A,Yadav S. Emergency obstetric hysterectomy: review at a tertiary care hospital Int $\mathbf{J}$ Reprod Contracept Obstet Gynecol.2016,5:3811-4

6. Babarinsa TA Hayman RG, Draycott TJ Secondary $\mathrm{PPH}$ challenges in evidence based causes and management. Eur J Obstet Gynecol Reprod Biol2011:159:25560

7. Medical News and Events. Fact sheet: Management of PPH.

8. Nanda S, Singhal S, Sharma D,Sood M, Singhal SK. Nonunion of utcrine incision: a rare case of secondary $\mathrm{PPH}$, report of two cases. Aust N ZJ Obest Gynaccol 1997:37:475-6. 